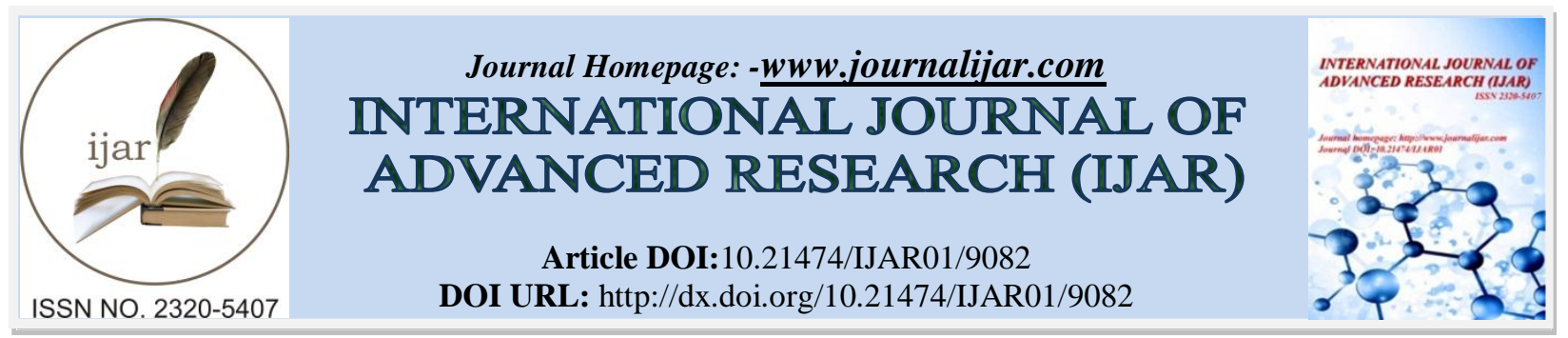

RESEARCH ARTICLE

\title{
HOUR-GLASS CONSTRICTION OF STOMACH - A CADAVERIC STUDY WITH SURGICAL EXPLANATION.
}

Naveena Swargam.

Associate Professor, Department of Anatomy, ESIC Medical College, Sanathnagar, Hyderabad, Telangana State, India- 500038.

\section{Manuscript Info}

Manuscript History

Received: 15 March 2019

Final Accepted: 17 April 2019

Published: May 2019

Key words:-

Hour-glass; Constriction; Cadaver; Adhesion.

\section{Abstract}

Background and Objectives: Hour-glass stomach can be described as a narrowing in the lumen of stomach due to contraction of its body wall. The aim of the present study is to relate the anatomical findings of hour glass stomach with their surgical significance.

Materials and Methods: Total 20 cadavers were dissected for the organ stomach, to observe the gross findings in a period of 3 consecutive academic years from 2016 to 2019, during routine dissection hours at ESIC Medical College, Sanathnagar, Hyderabad, Telangana State, India.

Results: In the present study there were 3 male cadavers, out of total 20 , aged between 55 to 65 years, having the stomach with a proper constriction in the body region giving the stomach a hour-glass appearance.

Conclusion: The main concern is to avoid the delay in treating the cause for hour-glass stomach, so that the consequent sufferings by the patient can be prevented.

Copy Right, IJAR, 2019,. All rights reserved.

\section{Introduction:-}

\section{Introduction}

Stomach occupies the epigastric, left hypochondriac and umbilical regions in the abdomen. It has fundus, body, pylorus, cardiac orifice, pyloric orifice, lesser curvature and greater curvature. Normally the shape of stomach is ' $J$ ', called sthenic type. Variant shapes of the stomach may be of congenital occurrence or acquired later on in life. The hour-glass stomach has one of the abnormal shapes, first described by an Italian anatomist Morgagni in the 18th century. The condition results due to the contracture of stomach wall usually in response to a gastric ulcer.

Gastric ulcers are found along the lesser curvature, but they may occur along the greater curvature of stomach near pyloric antrum. Opposite to the site of ulcer on the lesser curvature, a deep spasmodic notch is formed on the greater curvature. The ulcer usually shows extensive infiltration into the gastric wall. It has a great tendency to heal with the formation of scar tissue, which draws up the greater curvature towards the lesser curvature, causing an obstructive constriction with secondary dilatations. Congestion with oedema of the mucous membrane add to the obstruction followed by the spasm of muscular coat of the stomach. Perforation of a gastric ulcer may cause diffuse peritonitis leaving a broad band of adhesion round the stomach and adherent to the liver.

Corresponding Author:-Naveena Swargam.

Address:-Associate Professor, Department of Anatomy, ESIC Medical College, Sanathnagar, Hyderabad, 
The aim of the present study is to relate the anatomical findings of hour glass stomach with their surgical significance which would definitely help a surgeon in selecting a suitable surgical intervention with probable less complications.

\section{Materials and Methods:-}

Institutional Ethics Committee has approved as there is no objection to conduct the present study at ESIC Medical College, Sanathnagar, Hyderabad, Telangana State, India.

Total 20 adult human cadavers were dissected for the organ stomach, to observe the gross findings, in a period of 3 consecutive academic years from 2016 to 2019, during regular dissection hours for medical undergraduates. Routine abdominal dissection method was carried out to approach the stomach and its removal from the cadaver. All the stomach specimens were incised along the greater curvature to observe the interior.

Materials used were the cadaveric dissection instruments like Scalpel, Blunt Forceps, Pointed Forceps, Medium sized Scissors and Ligatures.

\section{Results:-}

In the present study there were 3 male cadavers, out of total 20, aged between 55 to 65 years, showed the stomach with a proper constriction in the body region giving it a typical hour-glass appearance. The observed gross findings of 3 hour-glass stomach specimens are elaborated below.

\section{Hour-glass stomach specimen 1}

The constriction was in the middle of the body of stomach, producing two almost equal sized pouches (Figure 1). On observation, interior of the stomach had no mucosal discoloration and no scar tissue was found. The annular constriction was occupying a narrow area of the stomach body wall where the wall has shown a proper thickness. There was no pyloric hypertrophy observed. There were no external bands or abnormal growth observed in and around the stomach wall.

\section{Hour-glass stomach specimen 2}

The constriction was in the middle of the body region of stomach, dividing it into two equal sized pouches (Figure 2). Interior of the stomach has shown mucosal discoloration in the upper pouch region. Wall of the stomach got thickened along the entire area of constriction (Figure 3). The annular constriction was observed to be occupying a larger area of stomach wall compared to what was observed in the specimen 1 . There was no hypertrophy in the pyloric region. There were no external bands or abnormal growth observed in and around the stomach wall.

\section{Hour-glass stomach specimen 3}

The constriction was near the pyloric antrum showing a lower smaller pyloric pouch and upper larger cardiac pouch (Figure 4). The annular constriction was observed to be occupying a larger area of stomach wall like what was observed in the specimen 2. Interior of the viscera was observed for any abnormal growth, mucosal discoloration and scar of an old healed ulcer. There was no abnormal growth and no scar tissue was found in the interior. Mucosal pigmentation was clearly observed in the area of constriction. There was a mild hypertrophy of muscular wall of the proximal part of antrum found at the annular constriction. There were no external peritoneal bands observed constricting the stomach wall.

\section{Discussion:-}

It is evident that the hour-glass stomach is usually produced by gross scarring around a severe chronic gastric ulcer.A constriction in the wall of the stomach leads to formation of secondary dilatations or pouches. Usually the ulcer causing the constriction is near the middle of the lesser curvature and the upper pouch is larger than the lower pouch. Occasionally the ulcer may be near the cardiac end of the stomach, so that the upper pouch is smaller than the lower one. Sometimes the ulcer may extend to the cardiac orifice and cause not only an hour-glass contraction but also oesophageal obstruction. Rarely an ulcer near the pylorus may cause an hour-glass contraction with a smaller lower pouch.

Nayak SB et al. reported a case of an atypical form of hour-glass stomach due to the presence of an unusual incisure at the greater curvature with the absence of evidence of any ulcer at the constriction and they concluded it as a 
congenital anomaly ${ }^{3}$. Occasionally, syphilis can cause hour-glass contraction. Carcinoma causes about $1 \%$ of hourglass contractions, in which the x-ray appearances differ much from those of simple hour-glass contractions ${ }^{4}$.

The annular constriction of stomach was found in 3 cases, out of 20 cadavers studied, in the present study with 15\% of frequency. Based on the gross features observed for all the 3 cases it was understood that the constriction might have been resulted due to the chronic healed gastric ulcer leaving a hypertrophied stomach wall musculature. C. Morrison Schroeder has reported a case of hourglass stomach caused by hypertrophy of the musculature proximal to the pyloric region which resembled carcinoma ${ }^{2}$. An atypical type of hourglass stomach was explained by S Prasanna et al. in their case report as a congenital anomaly ${ }^{5}$.

Method of treatment should be determined only after a careful examination of the conditions present and no routine method can be applicable to all cases of hour-glass stomach ${ }^{1}$. An ulcer of the stomach is frequently the seat of subsequent malignant growth, in such conditions partial gastrectomy is the only radical treatment where the open end of duodenum is closed and the jejunum is joined to the cardiac pouch. In the majority of cases gastroenterostomy between the cardiac pouch and the jejunum should be the operation of choice. This would be an efficient method of treatment where there is only a single constriction present without a pyloric stenosis and a double anastomosis connecting the jejunum with each pouch would be the method of choice, if pyloric stenosis exists.

\section{Conclusion:-}

A good deal in treating the hour-glass stomach depends upon the presence or absence of ulceration, pyloric obstruction and adhesions. The risk of operation is slight if the decision is taken before the obstruction is severe and the general condition of the patient is deteriorated. Whatever the form of operation undertaken, one should endeavour to carry out the procedure so as to satisfy two main objects, first, to remove the diseased portion of the stomach and secondly, to relieve the constriction in hour-glass condition, which will give the best prospect of the prevention of further sufferings from subsequent contraction along the line of suture. This well-recognized fact has great influence on choosing the surgical treatment and bringing an ultimate better prognosis.

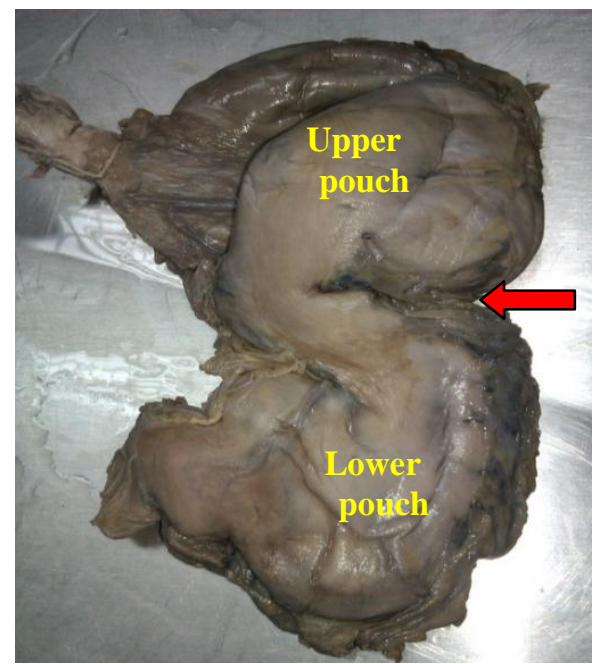

Fig 1:-Central constriction with equal sized pouches 


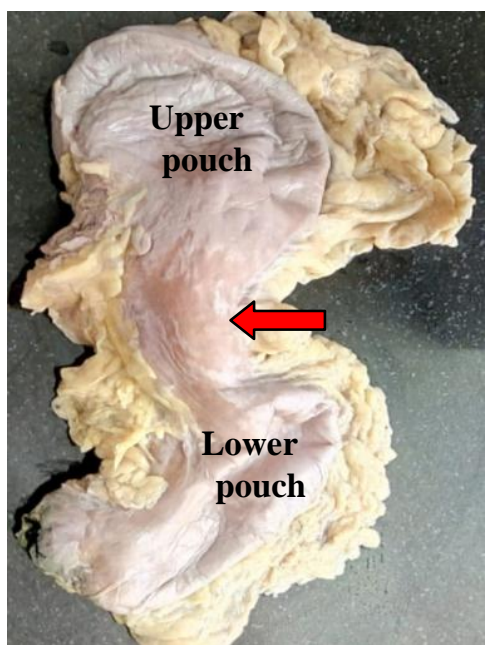

Fig 2:-Central constriction with equal sized pouches

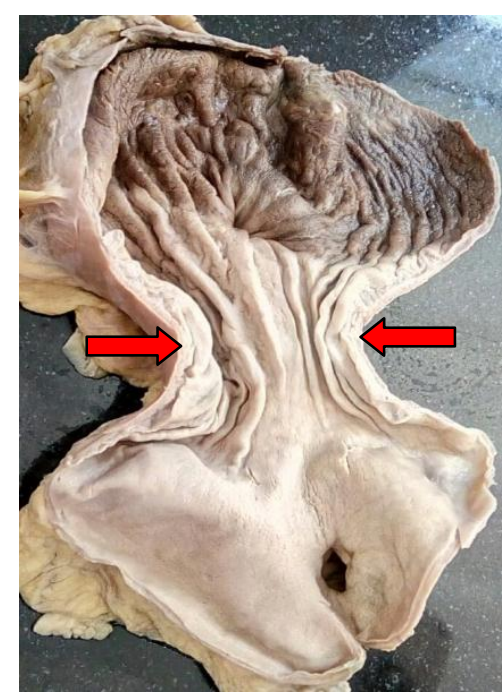

Fig 3:-Thickened stomach wall at the site of constriction

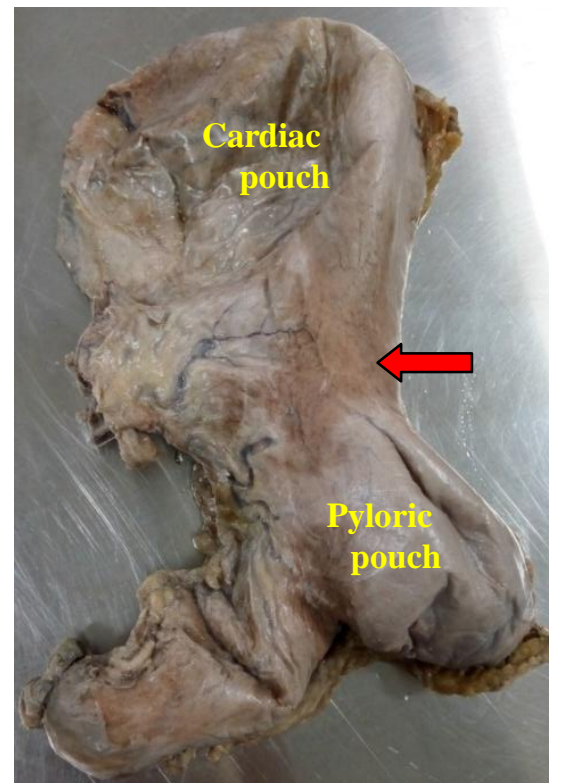

Fig 4:-Constriction with two unequal sized pouches 


\section{References:-}

1. A. Primrose (1914): Hour-glass contraction of the stomach with report of three cases. Canadian Medical Association Journal, 776-781.

2. C. Morrison Schroeder (1949): Hourglass stomach caused by annular muscular hypertrophy. Annals of Surgery. December, Volume 130, Number 6, 1085-1090.

3. Nayak SB, Kumar N, Bincy M, Mishra S, Rao SS, Shetty SD, Patil J (2017): An Atypical "Hourglass" Stomach Due to the Presence of an Unusual Incisure at the Greater Curvature. Online J Health Allied Scs. 16(1):13.

4. R.P. Rowlands (1931): Hour-glass contraction of the stomach. The British Medical Journal, July11, 50-52.

5. S. Prasanna et al (2018): Hourglass Stomach - A Case Report. IJAR Volume III Issue XI Nov - Dec, Page No: $1621-1623$. 